\title{
Comparative study between liver biopsy and non-invasive biomarkers in assessment of hepatic fibrosis in children with chronic liver diseases
}

\author{
Ola Galal Behairy ${ }^{1 *}$ (D), Ola Samir El-Shimi ${ }^{2}$ and Naglaa Hamed Shalan ${ }^{3}$
}

\begin{abstract}
Background: Liver biopsy is the gold standard for detecting the degree of liver fibrosis; however, invasiveness constitutes its main limiting factor in clinical application, so we aimed to evaluate the non-invasive biomarker formulas (APRI and FIB-4) and their modified forms by BMI z-score (M-APRI, M-FIB-4, and B-AST) compared to liver biopsy in the assessment of liver fibrosis in children with chronic liver diseases. Two hundred children aged $6.3 \pm$ 3.8 years (98 males, 102 females) with chronic liver diseases underwent liver biopsy. The stage of fibrosis was assessed according to the METAVIR system for all children, and the following non-invasive biomarker formulas were calculated: APRI, modified APRI (M-APRI: BMI z-score $\times$ APRI), Fibrosis-4 index (FIB-4), modified FIB-4 (M-FIB-4: BMI zscore $\times$ FIB-4), and B-AST (BMI $z$-score $\times$ AST). The best cutoff value was calculated to detect early fibrosis (F1-F2) from advanced liver fibrosis (F3-F4).

Results: There were positive correlations between all studied non-invasive biomarker models (APRI, FIB-4, M-APRI, M-FIB-4, B-AST) and fibrosis score as an increase in fibrosis score was associated with an increase in mean \pm SD of all studied biomarker formulas. The best cutoff values of non-invasive biomarker models in the diagnosis of early fibrosis (F1-F2) were APRI > 0.96, M-APRI > 0.16, FIB-4 >0.019, M-FIB-4 >0.005, and B-AST > -8 with an area under the curve above 0.7 each, while the best cutoff values of non-invasive biomarker models (APRI, M-APRI, FIB-4, M-FIB4 , and B-AST) in the diagnosis of advanced liver fibrosis (F3-F4) were $>1.96,>2.2,>0.045$, and $>0.015,>92.1$, respectively, with an area under the curve above 0.8 each.
\end{abstract}

Conclusion: APRI, M-APRI, FIB-4, M-FIB-4, and B-AST are good non-invasive alternatives to liver biopsy in the detection of liver fibrosis in children with chronic liver diseases of different etiologies especially those that include $\mathrm{BMI} z$-scores in their formulas.

Keywords: Chronic liver diseases, Children, Liver biopsy, Fibrosis, Non-invasive biomarkers

\section{Background}

The degree and progression of liver fibrosis are major determents of the outcome of liver disease in children. Liver biopsy is still the gold standard for diagnosis, grading, and staging of liver fibrosis [1]. However, liver

\footnotetext{
* Correspondence: osama.thorax@yahoo.com; ola.behery@fmed.bu.edu.eg

'Pediatrics Department, Faculty of Medicine, Benha University, Qualiopia,

Benha, Egypt

Full list of author information is available at the end of the article
}

biopsy is restricted being an invasive technique that might result in patient hazards such as pain, bleeding, and biliary system injury, along with variable accessibility, high cost, sampling mistakes, and inaccuracy due to inconsistency of pathologic interpretations [2]. These limitations promote the development of less invasive methods to assess liver fibrosis especially in children [3].

Hepatic fibrosis scores and serum biomarkers can serve as non-invasive, reproducible, and sensitive 
screening tools to predict fibrosis. Fibrosis scores such as aspartate aminotransferase/platelet ratio index (APRI) and fibrosis index based on four factors (Fibrosis-4 index, FIB-4) were developed based on the progression of liver pathology to cirrhosis and derived from the Apricot database in patients with chronic hepatitis $\mathrm{C}$ virus (HCV) infection [4]. Many of serum fibrosis models have been used to evaluate liver fibrosis and cirrhosis in adults with chronic hepatitis C. However, limited data on their accuracy in pediatric patients is available, and none of them has been fully validated in children [5]. Therefore, in this study, we aimed at the evaluation of non-invasive serum biomarker fibrosis models (APRI and FIB-4) and their modified forms by BMI $z$-score (M-APRI, M-FIB-4, and B-AST), compared to liver biopsy to assess liver fibrosis in children with chronic liver diseases.

\section{Methods}

\section{Subjects}

This was a 5-year observational cross-sectional study performed on 200 children suffering from various chronic liver diseases (a long-standing irreversible change in the hepatic structure that may end in complications like cirrhosis leading to premature death) [6], and their diagnosis was based on clinical, laboratory, and histopathological examination. Patients were recruited from the outpatient pediatric hepatology clinic between May 2015 and May 2019. Any patient with chronic liver disease of different etiologies (chronic viral hepatitis B or/and $C$, cholestatic liver disease, autoimmune hepatitis, and metabolic liver diseases) who underwent liver biopsy was included in the study. Patients were excluded if they had chronic liver disease with other co-morbidity as renal or C.V.S. or C.N.S. affection. This study was approved by the Ethical Committee of Faculty of Medicine, in accordance with "The Code of Ethics of the World Medical Association" (Declaration of Helsinki). Informed consent was obtained from parents prior to enrollment in the study after being fully informed about the study and its procedures.

\section{Methods}

- All enrolled children were subjected to the following:

- Full history taking and thorough clinical examination: with emphasis on clinical presentations (jaundice, abdominal pain and distension, melena and bleeding manifestations as hematuria, epistaxis, or bleeding gums), abdominal examination, and anthropometric measurements including weight, height, and BMI. Patients' weights were measured in kilograms (to the nearest $100 \mathrm{~g}$ ) using an electronic digital scale which accuracy was periodically verified using reference weights, while their heights were measured in centimeters (to the nearest millimeter). Children were measured on scales with height gauges, and the subject stood with back against the gauge and feet on the weighing platform. A software program that combines the raw data of the variables (age, sex, height, and weight) to compute a nutritional status index such as $z$-score, weight-forheight, weight-for-age, and height-for-age was used [7]. Patients' weights and heights were recorded on the day of the biomarker examination. BMI $z$-scores were calculated using the World Health Organization (WHO) Child Growth Standards and Growth Reference data with the WHO anthropometric calculator, AnthroPlus v.1.0.4.

Laboratory investigations: $9 \mathrm{ml}$ venous blood was drawn by aseptic venipuncture using a disposable sterile syringe. Blood was divided in different vacutainer tubes as follows: $3 \mathrm{ml}$ on K3-EDTA for CBC (CELL-DYN Emerald Hematology Analyzer, Abbott, IL, USA); $3 \mathrm{ml}$ on sodium-citrate for prothrombin time (PT), INR, and activated partial thromboplastin time (aPTT) (STA Compact Max, STAGO, France); and $3 \mathrm{ml}$ on the plain tube that was allowed to clot at room temperature for $30 \mathrm{~min}$ then serum was separated by centrifugation on $1262 \mathrm{~g}$ for $10 \mathrm{~min}$ for biochemical and serological testing including:

- Liver function tests: aspartate aminotransferase (AST), alanine aminotransferase (ALT), alkaline phosphatase (ALP), gamma-glutamyl transpeptidase (GGT), total and direct bilirubin, serum protein, and albumin (Biosystem A15 Auto-analyzer, BioSystem, Spain)

- Hepatitis B surface antigen (HBsAg) and anti-HCV antibody (HCV-Ab) by enzyme-linked immunosorbent assay (ELISA) were done and confirmation of $\mathrm{HCV}$ and HBV infection was done by using PCR (Biokit, Spain)

- Serum immunoglobulin G (IgG) by radial immunodiffusion (RID) (Binding Site, Birmingham, UK)

- Qualitative determination of anti-nuclear antibodies (ANA), anti-smooth muscle antibodies (ASMA), liver-kidney microsome antibodies (LKM-1), and anti-mitochondrial antibodies (AMA) by indirect immunofluorescence (IIF) (Nova Lite, INOVA diagnostics, USA)

- Work-up for Wilson's disease (serum ceruloplasmin, 24-h urinary copper before and after penicillamine) and Keyser-Fleischer ring 
Liver biopsy: Ultrasound-guided sample of living tissue of the liver was done for all patients either for diagnosis (when the above investigations were not enough for diagnosis) and/or detection of the degree of fibrosis using Menghini aspiration needle (Hepafix Luer Lock Braun Melsungen AG, Melsungen, Germany). A good enough core that contains at least five portal tracts was obtained. Biopsy specimens were fixed in formalin and deeply set within paraffin. Five-micrometer-length sections were cut and stained with hematoxylin and eosin to assess the histological activity of liver disease using the METAVIR scoring system [8] which comprises of five stages: F0 (no fibrosis), F1 (minimal fibrosis, portal fibrosis without septa), F2 (moderate fibrosis, portal fibrosis with few septa), F3 (severe fibrosis, sepal fibrosis with many septa but no cirrhosis), and F4 (cirrhosis). Sections were stained with Mason-Trichrome to assess fibrosis stage, Perls' Prussian blue stain to show iron deposition, and periodic acid chief stain to exclude alpha 1 anti-trypsin deficiency. Slides were blindly evaluated by two pathologists.

Non-invasive serum biomarker models for fibrosis evaluation: were calculated according to the published analytic recommendations $[9,10]$ as follows:

- Aspartate transaminase (AST)-to-platelet ratio index (APRI):

APRI $=[[$ AST $(I U / L) /($ AST ULN $(I U / L)] /$ platelet count $\left.\left(10^{9} / \mathrm{L}\right)\right] \times 100$

- Fibrosis-4 index (FIB-4): FIB- $4=[$ age $($ years $) \times$ AST $($ IU $/ L)] /[$ platelet count $\left.\left(10^{9} / \mathrm{L}\right) \times \sqrt{ } \mathrm{ALT}(\mathrm{IU} / \mathrm{L})\right]$

- The modified forms by BMI $z$-score: M-APRI $=$ APRI $\times$ BMI $z$-score M-FIB-4 = FIB- $4 \times$ BMI $z$-score $\mathrm{B}$-AST $=\mathrm{B}$-AST $(\mathrm{IU} / \mathrm{L}) \times$ BMI $z$-score

The upper limits of normal (ULN) for ALT and AST were established as $40 \mathrm{IU} / \mathrm{L}$, and the age used in formula and laboratory investigations was at the time of liver biopsy.

\section{Statistical methods}

The collected data were tabulated and analyzed using SPSS software, ver. 16 (SPSS Inc., Chicago, IL, USA) and MedCalc statistical software, ver. 17.2 (MedCalc Software, Mariakerke, Belgium). Categorical data were presented as numbers and percentages while quantitative data were expressed as mean \pm standard deviation, median, interquartile range (IQR), and range. Quantitative data were tested for normality using the KolmogorovSmirnov test, assuming normality at $P>0.05$. Differences between groups were tested using the KruskalWallis (KW) test for non-parametric variables; when significant, it was followed by post hoc multiple comparisons using the Bonferroni test to detect the significant pairs. Spearman's correlation coefficient (rho) was used to assess the correlation between non-parametric variables. ROC curve analysis was used to detect cutoff values for the studied markers with optimum sensitivity and specificity in the prediction of early fibrosis and advanced fibrosis. The accepted level of significance in this work was stated at $0.05(P<0.05$ was considered significant).

\section{Results}

This study was carried out on 200 children suffering from chronic liver disease of different etiologies; they were 98 male and 102 female, aged $6.3 \pm 3.8$ years. Forty percent of them had positive consanguinity and $30 \%$ had a family history of chronic liver disease. Among the studied patients, $25 \%$ were diagnosed with metabolic liver diseases (MLD) [glycogen storage disease 17\%, Niemann pick disease $2 \%$, Wilson disease $6 \%$ ], $44 \%$ were diagnosed with chronic hepatitis [autoimmune hepatitis $15 \%$, chronic hepatitis of unknown etiology $13 \%$, steatohepatitis $8 \%$, congenital hepatic fibrosis $8 \%$ ], $15 \%$ were diagnosed with viral hepatitis [HCV 10\%, HBV 5\%], and $16 \%$ had cholestasis disorders (progressive familial intrahepatic cholestasis 5\%, Alagille syndrome 6\%, alanti trypsin deficiency $1 \%$, Caroli's disease 2\%, DubinJohnson syndrome $2 \%$ ). The most clinical presentations of the studied hepatic patients were abdominal distension $(60 \%)$, jaundice $(44 \%)$, pallor $(21 \%)$, hepatomegaly (65\%), and splenomegaly (16\%). Anthropometric measurements are shown in Table 1.

The baseline data of liver function tests in the studied hepatic patients at the time of liver biopsy were found to be elevated above their upper limit of normal (ULN) (Table 1).

Histopathological evaluation of liver biopsy specimens revealed that the majority of the studied patients $(81 \%)$ showed mild disease activity. Regarding the degree of fibrosis, $41 \%$ had minimal fibrosis (F1), 19\% had moderate fibrosis (F2), and severe fibrosis (F3) was present in 9\% of patients (glycogen storage disease 5 patients, Wilson disease 4 patients, autoimmune hepatitis 5 patients, chronic hepatitis of unknown etiology 3 patients, $\alpha$ lanti trypsin deficiency 1 patient), while cirrhosis (F4) was observed in $9 \%$ of cases (congenital hepatic fibrosis 8 patients, chronic hepatitis of unknown etiology 4 patients, autoimmune hepatitis 6 patients ) (Table 2).

The median, interquartile range (IQR) of the five noninvasive serum biomarkers in our study group is shown in Table 3.

The studied non-invasive serum biomarkers (APRI, FIB-4, M-APRI, M-FIB-4, B-AST) showed significant positive correlations with HAI and fibrosis stage. The 
Table 1 Clinical and laboratory characteristics of the study group at the time of liver biopsy

\begin{tabular}{|c|c|c|}
\hline \multicolumn{2}{|c|}{ Variable } & \multirow{2}{*}{$\begin{array}{l}\text { Hepatic patients }(\boldsymbol{N}=\mathbf{2 0 0}) \\
6.3 \pm 3.8(2-15)\end{array}$} \\
\hline$\overline{\text { Age }}$ & & \\
\hline \multirow[t]{2}{*}{ Sex } & Male & 98 (49\%) \\
\hline & Female & $102(51 \%)$ \\
\hline \multicolumn{2}{|c|}{$\begin{array}{l}\text { Weight }(\mathbf{k g}) \\
\text { Centiles }\end{array}$} & $\begin{array}{l}18.2 \pm 5.9(9.0-40.0) \\
\text { (5th-75th) }\end{array}$ \\
\hline \multicolumn{2}{|c|}{$\begin{array}{l}\text { Height }(\mathrm{cm}) \\
\text { Centiles }\end{array}$} & $\begin{array}{l}95.4 \pm 32.4(70.0-149.0) \\
(<3 \text { rd-85th) }\end{array}$ \\
\hline \multicolumn{2}{|c|}{$\begin{array}{l}\text { BMI }\left(\mathrm{kg} / \mathrm{m}^{2}\right) \\
\text { Centiles }\end{array}$} & $\begin{array}{l}19.3 \pm 7.3(13.0-50.7) \\
(0.1 \text { th-95th) }\end{array}$ \\
\hline \multicolumn{2}{|c|}{ BMI z-score } & $0.13 \pm 2.9(-4.8: 6.0)$ \\
\hline \multicolumn{2}{|c|}{ AST (IU/L) } & $142.8 \pm 198.8(20-1080)$ \\
\hline \multicolumn{2}{|c|}{ ALT (IU/L) } & $155.7 \pm 205.8(18-1170)$ \\
\hline \multicolumn{2}{|c|}{ T. bilirubin (mg/dL) } & $4.5 \pm 5.03(0.2-21.0)$ \\
\hline \multicolumn{2}{|c|}{ D. bilirubin (mg/dL) } & $2.6 \pm 3.1(0.1-13.0)$ \\
\hline \multicolumn{2}{|c|}{ ALP (IU/L) } & $520.6 \pm 493(80-1192)$ \\
\hline \multicolumn{2}{|c|}{ GGT (IU/L) } & $73.7 \pm 119.5(41-668)$ \\
\hline \multicolumn{2}{|c|}{$\mathrm{Hb}(\mathrm{g} / \mathrm{dL})$} & $9.6 \pm 1.7(8.5-11.0)$ \\
\hline \multicolumn{2}{|c|}{ PLTs $\left(10^{3} / \mu \mathrm{L}\right)$} & $246.5 \pm 137.9(150.0-435.0)$ \\
\hline
\end{tabular}

Data expressed as mean \pm SD (range). BMI body mass index, AST aspartate aminotransferase, $A L T$ alanine transaminase, $T$. bilirubin total bilirubin, $D$. bilirubin direct bilirubin, ALP alkaline phosphatase, GGT gamma-glutamyl transferase, $\mathrm{Hb}$ hemoglobin, PLTs platelets

increase in the fibrosis score was associated with an increase in mean \pm SD of all five non-invasive biomarkers as the APRI score increases from $0.48 \pm 0.51$ in F0 to $8.11 \pm 52.9$ in F4, the FIB-4 score increases from $0.008 \pm$ 0.010 in F0 to $0.145 \pm 0.078$ in F4, the M-APRI score increases from $-2.03 \pm 2.88$ in F0 to $3.90 \pm 1.79$ in F4, MFIB- 4 increases from $-0.012 \pm 0.037$ in F0 to $0.56 \pm$

Table 2 Histopathological evaluation of the liver biopsy specimens among the study group according to METAVIR scoring system

\begin{tabular}{|c|c|c|c|c|}
\hline Variable & & & $\begin{array}{l}\text { Hepatic patients }(N \\
=200)\end{array}$ & $\begin{array}{l}\% \\
(100 \%)\end{array}$ \\
\hline \multirow[t]{5}{*}{ HAl } & $A 0=$ no activity & & 24 & $12.0 \%$ \\
\hline & A1 = mild activity & & 162 & $81.0 \%$ \\
\hline & $\begin{array}{l}\text { A2 = moderate } \\
\text { activity }\end{array}$ & & 8 & $4.0 \%$ \\
\hline & $\begin{array}{l}A 3=\text { severe } \\
\text { activity }\end{array}$ & & 6 & $3.0 \%$ \\
\hline & No fibrosis & FO & 44 & $22.0 \%$ \\
\hline \multirow{4}{*}{$\begin{array}{l}\text { Fibrosis } \\
\text { stages }\end{array}$} & Minimal fibrosis & $\mathrm{F} 1$ & 82 & $41.0 \%$ \\
\hline & Moderate fibrosis & $\mathrm{F} 2$ & 38 & $19.0 \%$ \\
\hline & Severe fibrosis & F3 & 18 & $9.0 \%$ \\
\hline & Cirrhosis & $\mathrm{F} 4$ & 18 & $9.0 \%$ \\
\hline
\end{tabular}

HAl histological activity index
Table 3 Non-invasive biomarkers in the study group

\begin{tabular}{lll}
\hline Non-invasive biomarkers & Median & Interquartile range )IQR) \\
\hline APRI & 0.6 & $0.24-1.7$ \\
FIB-4 & 0.01 & $0.04-0.05$ \\
M-APRI & 0.16 & $-0.64-0.65$ \\
M-FIB-4 & 0.001 & $-0.004-0.015$ \\
B-AST & 15.65 & $-84.4-64.5$ \\
\hline
\end{tabular}

APRI AST-platelet ratio index, FIB-4 Fibrosis-4 index, M-APRI modified APRI, $M$ FIB-4 modified FIB-4, B-AST AST in relation to BMI

0.071 in $\mathrm{F} 4$, and B-AST increases from $-213.4 \pm 369.8$ in F0 to $93.60 \pm 59.1$ in F4.

Moreover, APRI showed a significant positive correlation with liver enzymes (ALT, ALP, and GGT) and serum bilirubin (total and direct) levels, while M-APRI showed a significant positive correlation with age of patient and serum total bilirubin. The FIB- 4 index was found to be positively correlated with serum ALP level. There was a significant positive correlation between BAST and total bilirubin levels (Table 4).

In order to assess the performance of the studied noninvasive biomarkers in the detection of early fibrosis (F1-F2) and detection of advanced liver fibrosis (F3-F4), it was found that the best cutoff for APRI in the detection of early fibrosis (F1-F2) was > 0.96 with AUC 0.745 , sensitivity $46.4 \%$, and specificity $91.9 \%$, and in the detection of advanced liver fibrosis (F3-F4), the best cutoff point was $>1.96$, with AUC 0.849 , sensitivity $89.9 \%$, and specificity $84.5 \%$, while for M-APRI, the best cutoff for detection of early fibrosis (F1-F2) was $>0.16$ with AUC 0.831 , sensitivity $61.3 \%$, and specificity $96.5 \%$, and for the detection of advanced liver fibrosis (F2-F4), the cutoff point was $>2.2$ with AUC 0.958 , sensitivity $87.8 \%$, and specificity $96.6 \%$. In FIB-4, early fibrosis (F1F2) could be detected at a cutoff point $>0.019$ with AUC 0.750 , sensitivity $54.8 \%$, and specificity $94.5 \%$, while advanced liver fibrosis (F3-F4) could be detected at a cutoff point $>0.045$ with AUC 0.853 , sensitivity $78.8 \%$, and specificity $85.6 \%$. Using M-FIB-4, at a cutoff value $>0.005$, early fibrosis (F1-F2) could be detected with AUC 0.759 , sensitivity $49.7 \%$, and specificity $96.5 \%$, while at a cutoff value $>0.015$ with AUC 0.896 , sensitivity $89.8 \%$, and specificity $82.4 \%$, advanced liver fibrosis (F3F4) could be detected. The values of B-AST for detection of early fibrosis (F1-F2) was $>-8$ with AUC 0.758 , sensitivity $74.2 \%$, and specificity $75.7 \%$, while in the detection of advanced liver fibrosis (F3-F4), the cutoff point was $>92.1$, with AUC 0.955 , sensitivity $89.8 \%$, and specificity 91.2\% (Tables 5 and 6, Figs. 1 and 2).

\section{Discussion}

Liver biopsy is an approved procedure that is indicated to establish a diagnosis, assess the prognosis, and 
Table 4 Correlation between the non-invasive biomarkers and lab investigations and histopathological evaluation in the study group

\begin{tabular}{|c|c|c|c|c|c|c|c|c|c|c|}
\hline & \multicolumn{2}{|l|}{ APRI } & \multicolumn{2}{|l|}{ M-APRI } & \multicolumn{2}{|l|}{ FIB-4 } & \multicolumn{2}{|c|}{ M-FIB-4 } & \multicolumn{2}{|l|}{ B-AST } \\
\hline & Rho & $P$ & Rho & $P$ & Rho & $P$ & Rho & $P$ & Rho & $P$ \\
\hline Age (years) & 0.079 & 0.27 & 0.321 & 0.036 & - & - & - & - & 0.147 & 0.18 \\
\hline ALT (IU/L) & 0.649 & $<0.001$ & 0.018 & 0.85 & - & - & - & - & 0.018 & 0.85 \\
\hline T. bilirubin (mg/dL) & 0.297 & 0.003 & 0.235 & 0.018 & 0.156 & 0.12 & 0.189 & 0.059 & 0.223 & 0.026 \\
\hline D. bilirubin (mg/dL) & 0.314 & 0.002 & 0.186 & 0.67 & 0.173 & 0.08 & 0.167 & 0.098 & 0.160 & 0.11 \\
\hline ALP (IU/L) & 0.340 & 0.001 & 0.130 & 0.19 & 0.223 & 0.034 & 0.102 & 0.31 & 0.125 & 0.21 \\
\hline GGT (IU/L) & 0.253 & 0.011 & 0.107 & 0.29 & 0.179 & 0.074 & -0.063 & 0.63 & 0.08 & 0.37 \\
\hline $\mathrm{Hb}(\mathrm{g} / \mathrm{dL})$ & -0.007 & 0.94 & -0.076 & 0.45 & -0.05 & 0.62 & -0.135 & 0.18 & -0.136 & 0.18 \\
\hline HAI & 0.326 & 0.001 & 0.418 & $<0.001$ & 0.316 & 0.002 & 0.343 & $<0.001$ & 0.327 & 0.0013 \\
\hline Fibrosis stage & 0.568 & $<0.001$ & 0.629 & $<0.001$ & 0.473 & $<0.001$ & 0.537 & $<0.001$ & 0.473 & $<0.001$ \\
\hline
\end{tabular}

$B M I$ body mass index, $A L T$ alanine aminotransferase, $T$. bilirubin total bilirubin, D. bilirubin direct bilirubin, $A L P$ alkaline phosphatase, GGT gamma-glutamyl transferase, $H b$ hemoglobin, $H A l$ histological activity index

monitor therapy protocols [11]. Although the associated complications are infrequent, finding new alternative non-invasive methods for the evaluation of liver disease is of great interest [12]. In the current work, the majority of the studied 200 children with chronic liver disease had mild, moderate, and severe disease activity that were $81 \%, 4 \%$, and $3 \%$, respectively, while minimal, moderate, and severe fibrosis were $41 \%, 19 \%$, and $18 \%$, respectively. This comes in consistent with Behairy et al., who found that most of the studied $\mathrm{HCV}$ patients had minimal disease activity (80\%) and no/mild fibrosis $(72 \%)$; on the other hand, they found that the majority of the $\mathrm{AIH}$ group had mild to moderate activity (70\%) and moderate to severe fibrosis (95\%) and all Wilson disease group had mild to moderate activity (100\%) and moderate to severe fibrosis (100\%) [13] and Pokorska-Spiewak et al., who reported that most of the cases showed minimal to mild fibrosis [5]. However, Dhole et al. confirmed that advanced fibrosis appeared in $23 \%$ of cases [14]. Fibrosis staging in chronic liver disease is crucial in determining the prognosis, selecting patients fit for anti-fibrotic treatment, and monitoring treatment outcomes [15].

The median of the non-invasive biomarkers was APRI 0.6 , FIB-4 index 0.01, M-APRI 0.16, M-FIB-4 0.001, and B-AST 15.65. There were significant positive correlations between all of them and fibrosis score and HAI $(P$ $<0.05$ each), with a consistent increase in fibrosis score with the increase in mean \pm SD of the studied five noninvasive biomarkers. This comes in line with Abd ElGhaffar et al., who reported a highly significant positive correlation between APRI score and stage of fibrosis as assessed by the METAVIR scoring system $(r=0.53$ and $P$-value $=0.000)$. APRI score mean in patients was 0.71 \pm 0.48 and increased from $0.3 \pm 0.45$ in fibrosis stage $<2$ to $0.71 \pm 0.96$ in fibrosis stage $>2$ [16]. Also, de Ledinghen et al. studied APRI in comparison to Fibrosure and Fibroscan and found that APRI correlated with stages of fibrosis ( $\mathrm{rb}=0.32, P=0.03)$ [17].

The aspartate aminotransferase (AST) to platelet ratio index (APRI) is a simple biomarker that was developed based on the progression of liver pathology and includes standard-of-care tests that may reflect hepatocellular damage and early development of portal hypertension [10].

Furthermore, Leung et al. found that APRI performed better than FIB-4 in predicting fibrosis studied in children with cystic fibrosis liver disease. APRI may hold great promise for earlier detection of fibrosis or clinically silent liver disease in order to decrease further complications [18]. Similarly, Pokorska-Spiewak et al. found the mean \pm SD of the five biomarkers were APRI score 0.48 \pm 0.26 , FIB- $40.22 \pm 0.13$, M-APRI $0.28 \pm 0.69$, M-FIB- 4 $0.09 \pm 0.28$, and B-AST $31.71 \pm 69.87$ and confirmed a significant positive association between the fibrosis

Table 5 Performance of the studied non-invasive markers in the detection of early (F1-F2) in the studied group

\begin{tabular}{llllllll}
\hline & Cutoff value & Sens $\%$ & Spec\% & PPV\% & NPV\% & AUC & $95 \%$ CI \\
\hline APRI & $>0.96$ & $46.4 \%$ & $91.9 \%$ & $94.7 \%$ & $32.2 \%$ & 0.745 & $0.71-0.82$ \\
M-APRI & $>0.16$ & $61.3 \%$ & $96.5 \%$ & $97.9 \%$ & $40.4 \%$ & 0.831 & $0.63-0.88$ \\
FIB-4 & $>0.019$ & $54.8 \%$ & $94.5 \%$ & $97.7 \%$ & $36.9 \%$ & 0.750 & $0.55-0.85$ \\
M-FIB-4 & $>0.005$ & $49.7 \%$ & $96.5 \%$ & $97.4 \%$ & $34.4 \%$ & 0.759 & $0.64-0.79$ \\
B-AST & $>-8$ & $74.2 \%$ & $75.7 \%$ & $90.5 \%$ & $43.2 \%$ & 0.758 & $0.66-0.85$
\end{tabular}

Sens sensitivity, Spec specificity, PPV positive predictive value, NPV negative predictive value, AUC area under the curve, $C l$ confidence interval 
Table 6 Performance of the studied non-invasive biomarkers in the detection of advanced liver fibrosis (F3-F4) in the studied group

\begin{tabular}{llllllll}
\hline & Cutoff value & Sens $\%$ & Spec $\%$ & PPV\% & NPV\% & AUC & $95 \%$ Cl \\
\hline APRI & $>1.96$ & $89.9 \%$ & $84.5 \%$ & $34.8 \%$ & $98.7 \%$ & 0.849 & $0.76-0.92$ \\
M-APRI & $>2.2$ & $87.8 \%$ & $96.6 \%$ & $72.7 \%$ & $98.9 \%$ & 0.958 & $0.92-0.98$ \\
FIB-4 & $>0.045$ & $78.8 \%$ & $85.6 \%$ & $33.3 \%$ & $97.5 \%$ & 0.853 & $0.77-0.91$ \\
M-FIB-4 & $>0.015$ & $89.8 \%$ & $82.4 \%$ & $32 \%$ & $98.7 \%$ & 0.896 & $0.84-0.97$ \\
B-AST & $>92.1$ & $89.8 \%$ & $91.2 \%$ & $47.1 \%$ & $98.8 \%$ & 0.955 & $0.875-0.97$
\end{tabular}

Sens sensitivity, Spec specificity, PPV positive predictive value, NPV negative predictive value, AUC area under the curve, $C l$ confidence interval

stages and M-APRI and B-AST scores with a trend toward such an association with APRI and M-FIB-4 [5].

Elhenawy et al. stated that APRI and FIB-4 were significantly correlated with fibrosis in BA $(P=0.007)$ and were significantly higher in those with advanced fibrosis (Russo F4 and F5; $P=0.007$ ), and they stated that these non-invasive serological markers, which are derived from simple routine laboratory tests, may be of help in predicting advanced fibrosis and in long-term follow-up of infants with BA and minimize the need for repeated follow-up liver biopsies [19]. Many studies had reported a positive correlation between APRI score and degree of liver fibrosis [14, 17, 20].

Values of fibrosis biomarker models increase with the progression of fibrosis stages because they are dependent on ALT, AST, and platelet count, with ALT and AST increase and platelet count decrease with increasing of fibrosis. Various factors induce decreased platelet count such as secondary to decreased thrombopoietin production by hepatocytes and/or sequestration and destruction of platelets in the spleen when liver fibrosis advances and portal hypertension develops with age. However, with ongoing liver injury, AST release from mitochondria is increased and hepatic fibrosis decreases its clearance [21].

While assessing the performance of studied noninvasive biomarkers, we found that the best cutoff for APRI in the detection of early fibrosis (F1-F2) was $>0.96$ with an AUC of 0.745, while in advanced liver fibrosis (F3-F4), it was >1.96, with AUC 0.849. This comes in accordance with Kim et al. who reported that APRI AUROC for $F \geq 3$ and $F=4$ were 0.92 and 0.91 , respectively. Distinct optimal cutoff values of APRI for $F \geq 3$ and $F=4$ were obtained ( 1.01 and 1.41 , respectively) [20]. Also, de Ledinghen et al. found an AUC of 0.73 for predicting cirrhosis in children with various chronic liver

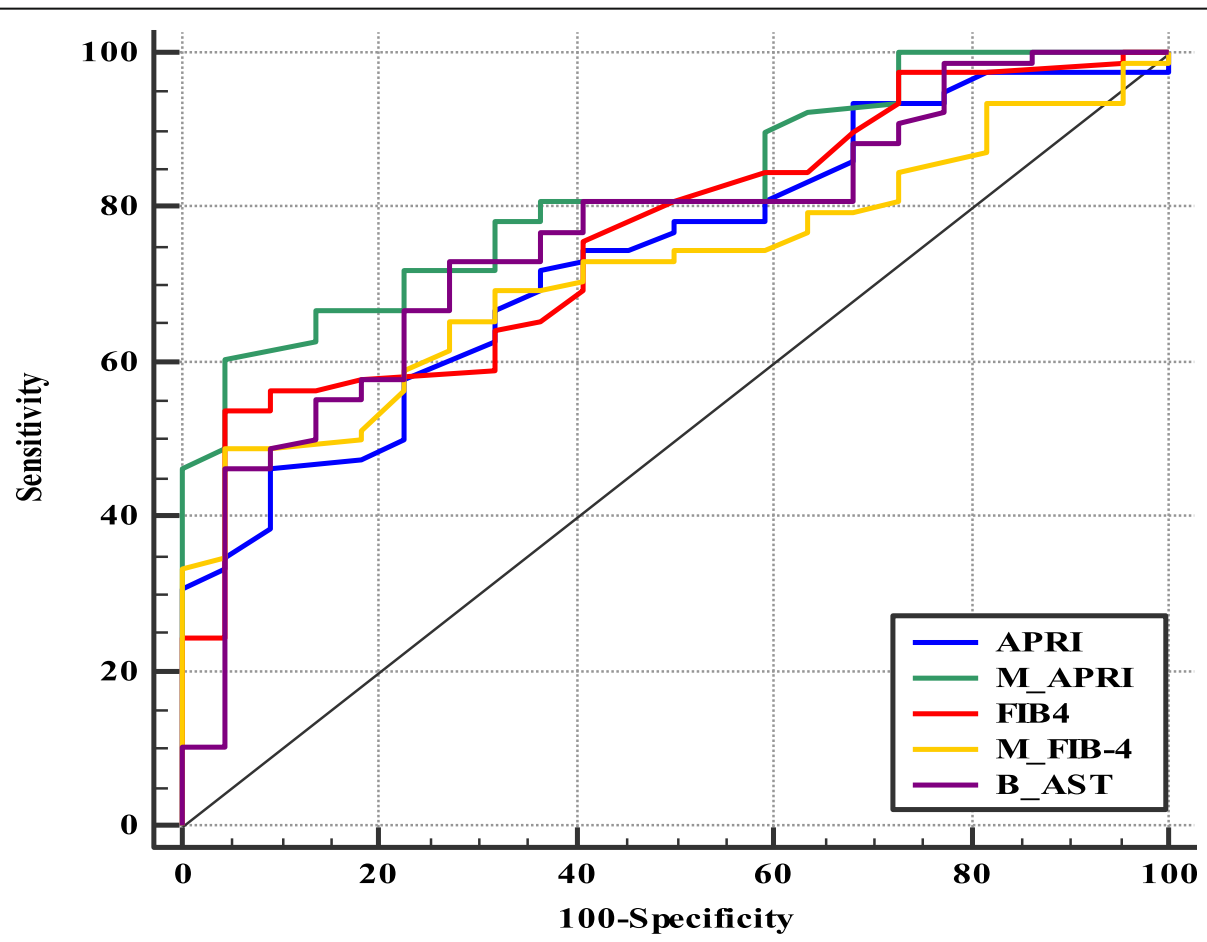

Fig. 1 Diagnostic performance (ROC curves) of the studied non-invasive biomarkers in the detection of early fibrosis (F1-F2) 


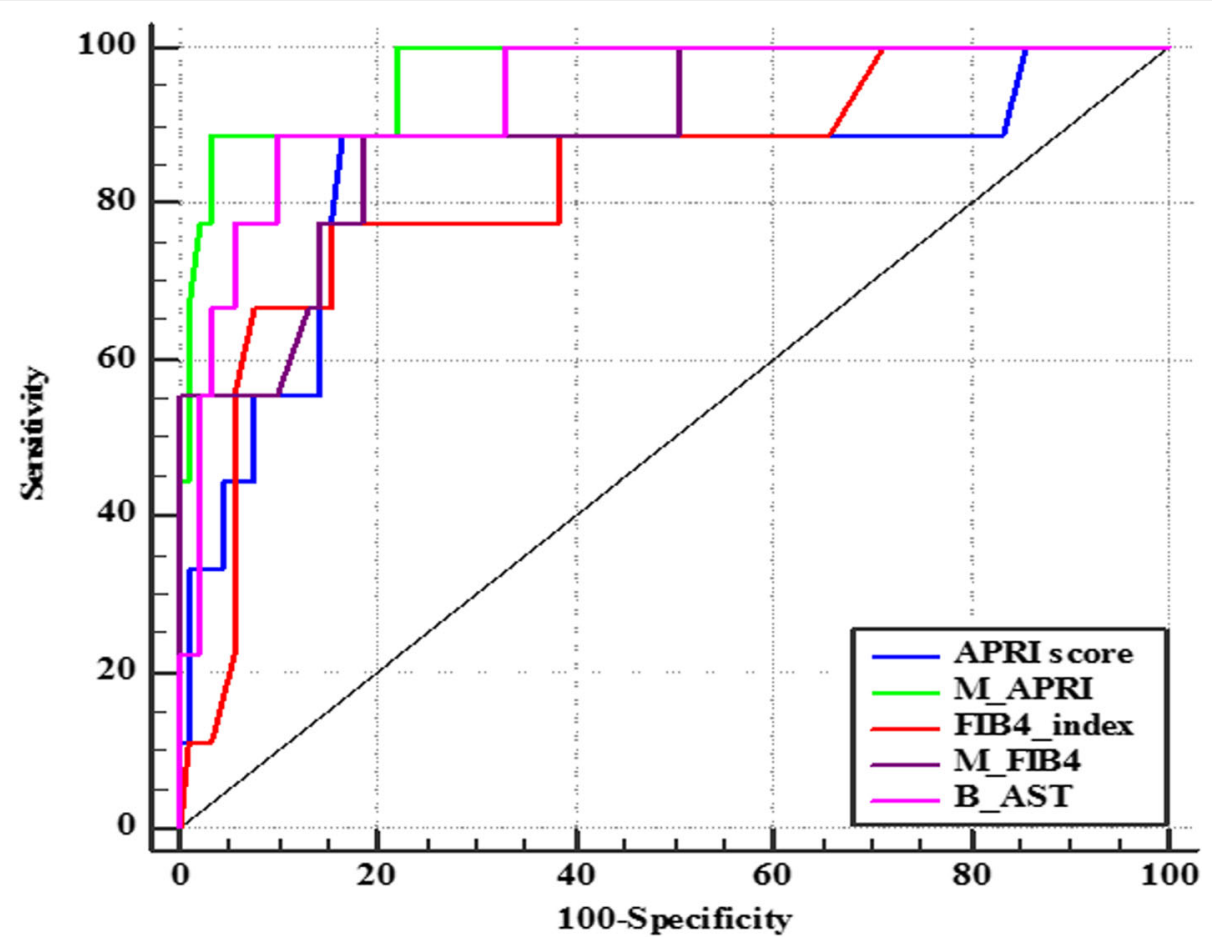

Fig. 2 Diagnostic performance (ROC curves) of the studied non-invasive biomarkers in the detection of advanced liver fibrosis (F3-F4)

diseases [17]. In addition, Grieve et al. using a cutoff value of 1.22 [AUC 0.83] showed a sensitivity of $75 \%$ and a specificity of $84 \%$ for macroscopic cirrhosis [22], and McGoogan et al. found that the APRI was moderately useful in predicting significant fibrosis where it could be a substitute for liver biopsy with AUC of 0.71 [23].

Moreover, in a meta-analysis that included 40 studies, it was shown that APRI score $>1.0$ had $76 \%$ sensitivity and $72 \%$ specificity for predicting cirrhosis. Additionally, APRI scores $>0.7$ had a $77 \%$ sensitivity and $72 \%$ specificity for predicting significant hepatic fibrosis [24].

Regarding the performance of FIB-4, the cutoff point to detect early fibrosis (F1-F2) was $>0.019$ with AUC 0.750, and to detect advanced fibrosis (F3-F4), it was $>0.045$ with AUC 0.853. These results agree with Elhenawy et al. reports which showed the AUC of FIB-4 was 0.0098 with AUR $0.644,61.9 \%$ sensitivity, and $61.9 \%$ specificity to discriminating advanced fibrosis [19]. More evidences came from Pokorska-Spiewak et al. study, where FIB-4 at cutoff point 0.18 , with AUR $0.708,85.7 \%$ sensitivity, and $93.7 \%$ $\mathrm{NPV}$, helped in detecting any stage of fibrosis; the cutoff point was 0.09 with AUC 0.586 [5]. On the other hand, Chen et al. reported that FIB-4 failed to correlate with the fibrosis stage. This may be due to the small number of patients in Chen's study $(n=24)$ [25].

In our study, during the assessment of the performance of BMI $z$-score modified models, we found M-
APRI, M-FIB-4, and B-AST at cutoff points $>0.16$, $>0.005$, and $>-8$, respectively with corresponding AUC $0.831,0.759$, and 0.758 , could detect early fibrosis (F1F2), while in detecting advanced fibrosis (F3-F4), the cutoff points of M-APRI, M-FIB-4, and B-AST were $>2.2,>0.015$, and $>92.1$ with AUC 0.958, 0.896, and 0.955 , respectively.

These results were in agreement with PokorskaSpiewak et al., who reported the cutoff values of MAPRI, M-FIB-4, and B-AST in the detection of any stage of fibrosis were $0.08,0.36$, and 0.56 with corresponding AUC 0.597, 0.568, and 0.614, respectively, while those for detection of moderate to severe fibrosis were 0.577, 0.179 , and 92.82 with corresponding AUC 0.842, 0.823, and 0.848 , respectively [5]. Also, Pokorska-Spiewak et al. suggest that liver fibrosis in children with $\mathrm{CHC}$ is positively associated with the BMI $z$-score. In both univariate and multivariate analyses, the BMI $z$-score was found to be an independent predictor of fibrosis among 42 pediatric patients with $\mathrm{CHC}(P=0.03)$ [12].

We had found that the BMI $z$-scores of modified noninvasive biomarker models (M-APRI, M-FIB-4, and BAST) had better diagnostic values in detecting early fibrosis (F1-F2) with AUC exceeded 0.7. Therefore, we supported the idea of incorporating the BMI $z$-score in the well-established APRI and FIB-4 non-invasive biomarker formulas to improve their performance in predicting advanced fibrosis. In addition, we confirmed the 
B-AST as a new non-invasive simple biomarker that can easily detect liver fibrosis using very simple parameters.

\section{Conclusion}

APRI, M-APRI, FIB-4, M-FIB-4, and B-AST are good non-invasive alternatives to liver biopsy in the detection of liver fibrosis and its extent in children with chronic liver diseases of different etiologies especially those that include BMI $z$-scores in their formulas.

\section{Acknowledgements}

The authors would like to thank all children and their parents who participated in this study.

\section{Authors' contributions}

1. OB contributed to the design and implementation of the research, aided in choosing the patients and helped shape the research, supervised the findings of this work, discussed the results, and read and approved the final manuscript.

2. OE contributed to the design and implementation of the research, aided in choosing the patients, performed the laboratory work and helped shape the research, supervised the findings of this work, discussed the results, and read and approved the final manuscript.

3. NS contributed to the design and implementation of the research, aided in choosing the patients and helped shape the research, supervised the findings of this work, discussed the results, read and approved the final manuscript.

"All authors have read and approved the manuscript."

\section{Funding}

No funding and no grants.

\section{Availability of data and materials}

All data generated or analyzed during this study are included in this published article.

\section{Declarations}

\section{Ethics approval and consent to participate}

The current study was approved by the Medical Research Ethical Committee of the Faculty of Medicine, Benha University. All subjects were informed about the procedures and the aim of the study, and informed written consent was obtained from the parents or caregivers of enrolled children. The committee's reference number is not available.

\section{Consent for publication}

Not applicable

\section{Competing interests}

The authors declare that they have no competing interests.

\section{Author details}

'Pediatrics Department, Faculty of Medicine, Benha University, Qualiopia, Benha, Egypt. ${ }^{2}$ Clinical Pathology Department, Faculty of Medicine, Benha University, Benha, Egypt. ${ }^{3}$ Pathology Department, Faculty of Medicine, Benha University, Benha, Egypt.

Received: 9 December 2019 Accepted: 13 September 2021 Published online: 25 October 2021

\section{References}

1. Hashmi SS, Bhatti AH, Malik MI, Rana A, Nasir H, Dar SF. et al (2017). Spectrum of histopathological diagnosis in pediatric patients with liver disorders in Pakistan. J Pak Med Assoc:; 67, (2):266-267.

2. Gaballah MA, Elsaid HH, Shaheen EN (2015) Enhanced liver fibrosis test can replace liver biopsy in detection of liver fibrosis in chronic hepatitis $C$ patients. International Journal of Advanced Research 3(4):145-150
3. Ruzman L, Mikolasevic I, Baraba Dekanic K, Milic S, Palcevski G (2018) Advances in diagnosis of chronic liver diseases in pediatric patients. World J Pediatr. 14(6):541-547. https://doi.org/10.1007/s12519-018-0197-8

4. Leung D.H (2017). Hepatic fibrosis scores and serum biomarkers in pediatric hepatology. Clinical Liver Disease; 9(5):125-130, DOI: https://doi. org/10.1002/cld.634.

5. Pokorska-Śpiewak M, Kowalik-Miko B, Aniszewska M, Magdalena P, Magdalena M (2017) Novel serum biomarkers modified by the body mass index z-score for the detection of liver fibrosis and steatosis in children with chronic hepatitis C. BMC Infectious Diseases 17(1):361-368. https://doi.org/1 $0.1186 /$ s12879-017-2462-1

6. Vergani GM, Vergani D (2002) Autoimmune liver disease. In Symposium on hepatology. Ind J Paediatr. 69(1):93-98. https://doi.org/10.1007/BF02723785

7. WHO Child Growth Standards based on length/height, weight and age (2006). Acta Pædiatrica; 450:76-85.

8. Bedossa P, Poynard T. (1996). An algorithm for the grading of activity in chronic hepatitis C. The METAVIR Cooperative Study Group. Hepatology, 24: 289-293. [PubMed: 8690394], 2, DOI: https://doi.org/10.1002/hep.510240201

9. Sterling RK, Lissen E, Clumeck N, Sola R, Correa MC, Montaner J, S. Sulkowski M, Torriani FJ, Dieterich DT, Thomas DL, Messinger D, Nelson M, APRICOT Clinical Investigators (2006) Development of a simple noninvasive index to predict significant fibrosis in patients with HIV/HCV coinfection. Hepatology 43(6):1317-1325. https://doi.org/10.1002/hep.21178

10. Wai CT, Greenson JK, Fontana RJ, Kalbfleisch JD, Marrero JA, Conjeevaram HS, Lok AS (2003) A simple noninvasive index can predict both significant fibrosis and cirrhosis in patients with chronic hepatitis C. Hepatology 38(2): 518-526. https://doi.org/10.1053/jhep.2003.50346

11. Sparchez Z (2005) Complications after percutaneous liver biopsy in diffuse hepatopathies. Rom J Gastroenterol. 14(4):379-384

12. Pokorska-Śpiewak M, Kowalik-Mikołajewska B, Aniszewska M, Pluta M, Marczyńska M (2015) Is liver biopsy still needed in children with chronic viral hepatitis? World J Gastroenterol. 21(42):12141-12149. https://doi.org/1 0.3748/wjg.v21.i42.12141

13. Behairy B, Sira MM, Zalata KR, Salama E, Abdallah MA (2016) Transient elastography compared to liver biopsy and morphometry for predicting fibrosis in pediatric chronic liver disease; does etiology matter? World Journal of gastroenterology 22(16):4249-4238. https://doi.org/10.3748/wjg. v22.i16.4238

14. Dhole SD, Kher AS, Ghildiyal RG, Tambse MP (2015) Chronic liver diseases in children: clinical profile and histology. Journal of Clinical and Diagnostic Research 9(7):4-7

15. Stauber RE, Lackner C (2007) Noninvasive diagnosis of hepatic fibrosis in chronic hepatitis C. World Journal of Gastroenterology 13(32):4287-4294. https://doi.org/10.3748/wjg.v13.i32.4287

16. Abdel Ghaffar T, Youssef A, Zalata K, Aisha ElSharkawy A, Mowafy M, Wanis A et al (2016) Non-invasive assessment of liver fibrosis in Egyptian children with chronic liver diseases. Curr Pediatr Res 20(1\&2):57-63

17. de Ledinghen V, Le Bail B, Rebouissoux L, Fournier C, Foucher J, Miette V et al (2007) Liver stiffness measurement in children using FibroScan: feasibility study and comparison with Fibrotest, aspartate transaminase to platelets ratio index, and liver biopsy. J Pediatr Gastroenterol Nutr 45(4):443450. https://doi.org/10.1097/MPG.0b013e31812e56ff

18. Leung DH, Khan M, Minard CG, Guffey D, Ramm LE, Clouston AD, Miller G, Lewindon PJ, Shepherd RW, Ramm GA (2015) Aspartate aminotransferase to platelet ratio and fibrosis-4 as biomarkers in biopsy-validated pediatric cystic fibrosis liver disease. Hepatology 62(5):1576-1583. https://doi.org/10.1002/ hep.28016

19. Elhenawy IA, Ghanem HS, Sultan MM, Sira MM (2017) Utility of noninvasive serum biomarkers of liver fibrosis in infants with biliary atresia. Adv Res Gastroentero Hepatol 3(2):555607

20. Kim SY, Seok JY, Han SJ, Koh H (2010) Assessment of liver fibrosis and cirrhosis by aspartate amino transferase-to-platelet ratio index in children with biliary atresia. J Pediatr Gastroenterol Nutr 51(2):198-202. https://doi. org/10.1097/MPG.0b013e3181da1d98

21. Khairy M, Abdel-Rahman M, El-Raziky M, El-Akel W, Zayed N, Hany Khatab H et al (2012) Non-invasive prediction of hepatic fibrosis in patients with chronic HCV based on the routine pre-treatment workup. Hepat Mon:12

22. Grieve A, Makin E, Davenport M (2013) Aspartate aminotransferase-toplatelet ratio index (APRi) in infants with biliary atresia: prognostic value at presentation. J Ped Surg 48(4):789-795. https://doi.org/10.1016/j.jpedsurg.2 012.10.010 
23. McGoogan KE, Smith PB, Choi SS, Berman W, Jhaveri R (2010) Performance of the AST-to-platelet ratio index as a noninvasive marker of fibrosis in pediatric patients with chronic viral hepatitis. Journal of pediatric gastroenterology and nutrition 50(3):344-346. https://doi.org/10.1097/MPG Ob013e3181aed725

24. Lin ZH, Xin YN, Dong QJ, Wang Q, Jiang XJ, Zhan SH, Sun Y, Xuan SY (2011) Performance of the aspartate aminotransferase-to-platelet ratio index for the staging of hepatitis C-related fibrosis: an updated meta-analysis. Hepatology 53(3):726-736. https://doi.org/10.1002/hep.24105

25. Chen S, Liao B, Zhong Z, Zheng Y, Liu B, Shan Q, Xie X, Zhou L (2016) Supersonic shearwave elastography in the assessment of liver fibrosis for postoperative patients with biliary atresia. Sci Rep 6(1). https://doi.org/10.1 038/srep31057

\section{Publisher's Note}

Springer Nature remains neutral with regard to jurisdictional claims in published maps and institutional affiliations.

\section{Submit your manuscript to a SpringerOpen ${ }^{\circ}$ journal and benefit from:}

- Convenient online submission

- Rigorous peer review

- Open access: articles freely available online

High visibility within the field

- Retaining the copyright to your article

Submit your next manuscript at $\boldsymbol{\nabla}$ springeropen.com 